\title{
EI dialecto: estigma y obstáculo en la recepción del Wiener Volkstheater
}

\author{
Juan Antonio AlBALADEJO MARTíNEZ \\ Universidad de Alicante \\ Ja.albaladejo@ua.es
}

Recibido: 8 de diciembre de 2010

Aceptado: 1 de marzo de 2011

\begin{abstract}
RESUMEN
El gran reconocimiento del que goza el teatro popular vienés en Austria, especialmente las obras de Johann Nestroy y Ferdinand Raimund, contrasta con una apreciación mucho menos favorable en la vecina Alemania, sobre todo si se comparan las historias de la literatura escritas a ambos lados de la frontera. Una de las razones que ha contribuido a limitar la recepción de esta literatura es el empleo del dialecto local. Éste se introduce como recurso lingüístico que crea, en el seno del texto, contrastes respecto de la lengua estándar. El uso del dialecto ha llevado a que el teatro popular vienés a menudo haya sido clasificado, erróneamente, como literatura dialectal. El análisis pormenorizado de la realización lingüística del lenguaje popular, presente en las obras, demuestra que se trata de textos dialectalmente marcados. La presencia de rasgos dialectales ni impide la comprensibilidad suprarregional de estos textos ni justifica el confinamiento local de una literatura cuyo valor estético justificaría su inclusión en el canon de la literatura universal.
\end{abstract}

Palabras clave: Literatura, teatro popular, recepción, dialecto, marcación, canon literario.

\section{The Vernacular: Stigma and Obstacle to the Reception of the Viennese Popular Theatre}

\begin{abstract}
The Viennese Popular Theatre enjoys a widespread recognition in its native Austria (especially the works of Johann Nestroy and Ferdinand Raimund), thus contrasting with a far less favorable appreciation in the neighboring Germany. This is especially true when one compares the histories of literature written on both sides of the border. One of the reasons that favours the limitation of the reception of this literature is the use of the local vernacular. It is brought in as a linguistic resource that creates contrasts with the standard language within the texts. The presence of the vernacular has often fostered the error of classifying the Viennese Popular Theatre as vernacular literature. A detailed analysis of the linguistic expression of the popular language, featured in the works, shows that these are dialectally marked texts. The presence of dialectal features does neither prevent the supraregional understandability of these texts nor justify the local confinement of a literature with an aesthetic value that would justify its incorporation into the canon of world literature.
\end{abstract}

Keywords: Literature, Popular Theatre, Reception, Vernacular, Marking, Literary Canon.

SUMARIO: 1. Introducción. 2. Origen e historia del Wiener Volkstheater. 3. El dialecto en la literatura. 3.1. Uso y función del dialecto en el texto literario. 3.2. El dialecto en el Wiener Volkstheater. 3.3. Identificación errónea con la literatura dialectal. 4. Conclusiones. 


\section{Introducción}

Cuando en el presente artículo se habla de Wiener Volkstheater nos referimos, esencialmente, a la obra de los dos representantes más destacados de esta literatura: Johann Nestroy y Ferdinand Raimund. Su producción artística se desarrolla durante las épocas del Biedermeier y Vormärz (en la última etapa, en Nestroy se percibe la transición hacia formas más realistas). Es precisamente en esta fase, aproximadamente entre 1820 y 1850 , en la que el teatro popular vienés adquiere auténtico valor literario ${ }^{1}$.

Mientras que, a lo largo del siglo XX, los textos de Nestroy y Raimund han logrado entrar en el canon nacional de su Austria natal ${ }^{2}$, en otros países, incluidos los de lengua alemana, este hecho aún no se ha producido. Que unos textos pasen de la periferia al centro del polisistema ${ }^{3}$ literario demuestra la historicidad y dependencia sociocultural del canon ${ }^{4}$ :

$\breve{S S k l o v s k i j}^{5}$ scheint der Erste gewesen zu sein, der soziokulturelle Unterschiede in der Textproduktion unter dem Aspekt der literarischen Stratifikation beschrieben hat. Ihm zufolge [...] werden in der Literatur bestimmte Eigenschaften kanonisiert, andere dagegen nicht. Hierbei sind unter "kanonisiert" die literarischen Normen und Werke (d. h. sowohl Modelle als auch Texte) zu verstehen, die von den dominanten Kreisen einer Kultur als legitim akzeptiert werden und deren sichtbarste Produkte Teil des historischen Erbes der Gemeinschaft werden. Im Gegensatz dazu bezieht sich "nicht kanonisiert" auf die von den dominanten Kreisen als illegitim abgelehnten Normen und Werke, die in der Gemeinschaft auf lange Sicht oft in Vergessenheit geraten (sofern kein Statuswechsel stattfindet).

${ }^{1}$ HeIN (1997: 107): "Die Zeit zwischen dem Wiener Kongress und der bürgerlichen Revolution von 1848 wird von vielen als wichtigste Phase des Wiener Volkstheaters bezeichnet [...]".

${ }^{2}$ Existen unas sociedades dedicadas al estudio y difusión de sus obras: la Internationale Nestroy Gesellschaft (publica una revista, Nestroyana, y celebra periódicamente reuniones científicas) y la Raimund-Gesellschaft, que desde hace más de setenta años promueve la producción literaria del otro gran representante del Wiener Volkstheater. La veneración que en Austria se siente por Nestroy ha llevado a que se le diera el apodo de "Shakespeare austriaco".

${ }^{3}$ Nos estamos refiriendo a la teoría del polisistema, desarrollada por Itamar Even-Zohar, que puede describirse de la siguiente forma: "Die Bezeichnung Polysystem soll auf die Heterogenität und diachronische Dynamik des Literatur(poly)systems aufmerksam machen. Jede Literatur ist Teil eines größeren Polysystems, nämlich der Gesamtkultur der betreffenden Gemeinschaft. Zwischen den verschiedenen Polysystemen, die diese Gesamtkultur bilden - also z. B. Literatur, Sprache, Wirtschaft, Politik -, bestehen komplexe Korrelationen. Die Gesamtkultur ihrerseits gehört zu einem noch größeren Makro-Polysystem, das die Kultur mehrerer Gemeinschaften umfasst. Die Grenzen zwischen den verschiedenen Polysystemen sind fließend und verschieben sich kontinuierlich. Ziel der Polysystemtheorie ist die Entdeckung von Gesetzen, mit denen sich die Bewegung zwischen Zentren und Peripherien innerhalb eines Polysystems oder zwischen verschiedenen Polysystemen und somit der diachronische Wandel - beschreiben lassen" (HAGEMANN 2009: 10-11).

${ }^{4}$ Lo mismo sucede con la concepción estética, como bien resalta ECO (2007:10): "Los conceptos de bello y de feo están en relación con los distintos períodos históricos o las distintas culturas [...]".

${ }^{5}$ Mantenemos la grafía divergente del nombre de este autor, tal y como aparece en las distintas obras citadas en el presente trabajo: Šklovskij / Šklovski. 
Kanonizität ist daher kein auf irgendeiner Ebene den Textaktivitäten innewohnendes Merkmal; sie ist kein Euphemismus für "gute" Literatur im Gegensatz zu "schlechter" (Even-Zohar 2009: 46).

Nestroy tardó más de medio siglo, tras su muerte, en convertirse en "burgtheaterwürdig", casi un siglo después de que comenzara a triunfar sobre los escenarios del Volkstheater. Es la señal más visible del cambio de actitud de los círculos dominantes del mundo de la literatura en Austria frente a su obra.

A pesar de que también en Alemania y Suiza se siguen representando numerosas piezas de los mencionados autores, el éxito de público que cosechan no se corresponde con la menor atención y valoración por parte del mundo de la investigación y crítica literarias ${ }^{7}$. En estas esferas no se ha producido el cambio de estatus de la obra de los escritores más destacados del teatro popular vienés. La diferencia de apreciación también se plasma en las historias de la literatura, dependiendo de los ámbitos en y para los que se elaboran. Así, frente al lugar destacado que ocupan Nestroy y Raimund en las obras de referencia austriacas, en las de la República federal no suelen superar la consideración de meros comparsas ${ }^{8}$.

En nuestra opinión, son varios los factores que explican la tendencia patente de minimizar la relevancia de unas creaciones artísticas que, por su valor intrínseco, deberían trascender del ámbito nacional y formar parte del canon literario universal.

Sin duda, la cuestión genérica ${ }^{9}$ ha contribuido, en gran medida, a conformar prejuicios estéticos no del todo favorables. Junto a ésta, otro aspecto importante que ha determinado la opinión de investigadores y críticos literarios es la elaboración lingüística. A partir del estilo (humilis) de escritura y del lenguaje predominante, marcado por rasgos dialectales, se infiere, equivocadamente, un carácter exclusivamente local o regional de las piezas ${ }^{10}$.

\footnotetext{
${ }^{6}$ Es decir, digno de ser representado en el Burgtheater, el teatro más prestigioso en lengua alemana y templo de la alta literatura y cultura.

${ }^{7}$ Como indica HeIN:"Die Forschung hat das Wiener Volkstheater freilich eher stiefmütterlich behandelt und z.T. in den Bereich des Vor- oder Nicht-Literarischen bzw. der trivialen Unterhaltung abgeschoben" (1997: VII).

${ }^{8}$ Excepto las historias de la literatura que se elaboran en Austria, tales como la Literaturgeschichte Österreichs. Von den Anfängen im Mittelalter bis zur Gegenwart de Herbert Zeman. En la Historia de la literatura alemana de Wolfgang Beutin et. al. (versión española publicada en Cátedra, 1991), uno de los manuales de referencia de los germanistas españoles, no se hace ninguna mención del Wiener Volkstheater. En la Historia de la literatura alemana de Hans Gerd Roetzer y Marisa Siguán, por el contrario, se menciona el teatro popular vienés en relación con la literatura Biedermeier y se analizan brevemente dos obras de Raimund y Nestroy, respectivamente.

${ }^{9}$ El género más representativo en la obra de Nestroy, junto con la pieza mágica, es la farsa. Se trata de un género literario con unos rasgos característicos contrarios a los de la "alta" literatura. WILPERT (1989: 701) la define como: "Anspruchslose, volkstümliche Komödienform, die durch vordergründige, primitiv-derbe und in der Übertreibung die Grenzen des Wahrscheinlichen überschreitende niedere Komik nicht nur Lächeln, sondern Lachen erregen will [...]”.

${ }^{10}$ TRAMER (1949: III): "Ferdinand Raimund may well be regarded as one of the outstanding figures of the continental theater for his singular histrionic achievements and for his genius in creating dramatic fairy tales. And yet his great name is almost entirely unknown in the English-speaking
} 
La recepción de los textos más allá de las fronteras nacionales se ha visto entorpecida por una cierta dificultad lingüística más aparente que real. Esto se ha manifestado sobre todo a nivel interlingüístico ${ }^{11}$. No obstante, también en el ámbito de la lengua alemana la recepción se ha visto limitada por la habitual ausencia de un análisis profundo de la variación del lenguaje utilizado por los autores del teatro popular vienés. Todo esto ha propiciado la clasificación errónea de los textos. La presencia de rasgos y marcas dialectales ya suele motivar por sí sola la calificación de ese lenguaje como "dialectal", algo que no se justifica en el caso de los textos de Nestroy y Raimund ${ }^{12}$. Esa calificación, más impresionista que científica, no responde en absoluto a la realidad textual, caracterizada por una composición lingüística de gran complejidad que no es posible reducir a una simple oposición "dialecto-lengua estándar". Si el lenguaje marcado de los textos se identifica con el dialecto, sin que se realice una mayor diferenciación, es comprensible que este error de apreciación desemboque en una clasificación equívoca de los textos del Volkstheater como literatura dialectal. Wilpert (1989: 181-182) incluye en la siguiente definición de Mundartdichtung el Wiener Volkstheater:

Vollständig in Mundart verfaßte Dichtung im Gegensatz zur Dichtung in der Hochoder Schriftsprache. Vor Entstehen einer solchen ist praktisch jede Dichtung an eine bestimmte Mundart gebunden. [...]. Im allgemeinen sind in der Dialektdichtung alle Gattungen vertreten, doch ist mangels einheitlicher Orthographie die Wirkung bei mündlichem Vortrag am größten und die Reichweite meist auf den Mundartraum beschränkt [...]. Der Schwabe S. Sailer (1714-77), der Oberfranke K. Grübel (17361809, Gedichte in Nürnberger Mundart, 1798) sowie J. H. Voss (plattdt.), J. G. D. Arnold (els.) und J. M. Usteri (schweiz.) bringen die ersten Ansätze einer neuen Dialektdichtung. J. P. Hebels Alemannische Gedichte (1803) dringen über die Grenzen der Mundart hinaus vor und regen weitere Dialektdichtung an, während in den Volkstheatern der Alpenländer das Dialektstück weitgehend in Geltung geblieben war (Stranitzky, Castelli, Raimund, Nestroy bis Anzengruber) und im 20. Jh. sich erneuert (Ö. v. Horváth, W. Bauer). Im 19. Jh. tritt sodann insbesondere der plattdeutsche Raum mit Dialektdichtung von geradezu klassischer Geltung in Lyrik (Klaus Groth) und Erzählkunst (Fritz Reuter, R. Kinau) hervor.

world so familiar with authors of lesser significance. For this odd situation a literary prejudice may be chiefly responsible: the claim that Raimund's works are nothing more than 'plays of Vienna folk life', of such as to be merely of local or regional interest. But in all of Raimund's plays there are only a few scenes that are laid in Vienna. Though inspired by and written in the spirit of the local comedy theater of that epoch, they all deal with universal human themes, with good and evil, as good fairy tales commonly do".

11 " [...] the greatest obstacle to success Nestroy faced -and still faces- is that of language. In a century when much was made over the purity of the mother tongue, one can imagine the reception given his heady brew of literary German and the Viennese dialect, heavily laced with puns, double entendres, portmanteau words, foreign phrases, mixed metaphors, neologisms, proverbs taken out of context, virtually unpronounceable tongue-twisters, etc." (HARRISON / WILSON 1986: 6).

${ }^{12}$ Algo que se podrá comprobar en el capítulo dedicado al análisis lingüístico del elemento dialectal en esta literatura. 
Aquí se están englobando, bajo la etiqueta de literatura dialectal, una serie de autores cuyos textos muestran rasgos lingüísticos claramente divergentes. De esta manera, mientras que los textos, por ejemplo, de Klaus Groth, Fritz Reuter, J. P. Hebel o Ludwig Anzengruber realmente pertenecen a la literatura dialectal ${ }^{13}$, no ocurre lo mismo con las obras de Nestroy y Raimund (y mucho menos las de Horváth $\left.^{14}\right)$. Para ilustrar las considerables diferencias respecto del tratamiento lingüístico de unos y otros, ofrecemos a continuación unas muestras representativas:

Döfft bün ick ok un heww ok Pädings hatt: vir Stück. Un wenn min vir Pädings noch lewten un güngen mit mi äwer de Strat, denn würden de Lüd' still stahn un seggen: "Kikt, wat sünd dat för dägte Kirls! Nah so'n Ort kann ein up Stun'ns lang säuken; dat sünd noch Pädings!" Un ein was dorunner, de was en Kopp länger as de annern un kek äwer ehr rut as Saul äwer sine Bräuder; dat was de oll Amtshauptmann Wewer un hadd en saubern blagen Rock an un 'ne gelrige Hos' un lange blankgewichste Stäweln, un was sin Gesicht ok von Pocken terreten un hadd de Düwel ok sin Arwten dorup döscht, dat hei utsach, as hadd hei mit dat Gesicht up en Ruhrstaul seten (Reuter 1886: 39).

Er schlooft; er schlooft! Do lyt er, wie ne Groof!

Du lieben Engel, was i bitt,

by Liib un Lebe verwach mer nit!

Gott gitt's de Synen im Schloof!

Verwach mer nit, verwach mer nit!

Dy Muetter goht mit stillem Tritt,

si goht mit zartem Muettersinn

Un holt e Baum im Chämmerli dinn. [...] (Hebel 1978: 28).

LORENZ.- Was schaffen S'?

WURZEL.- Wo steckst denn, daß dich um mich nicht umschaust?

LORENZ.- Grad bin ich hinausgangen. Die Fräulein Lottel war vorher da und hat mit Ihnen reden wollen.

WURZEL.- Untersteh dich nicht, daß du ein Wort von ihr redst, ich will nichts wissen von der Wasserprinzessin.

Ist das ein Betragen für ein Haus wie das meinige? Statt daß ein vampirenes Kleid anleget und mit ihren Vatern auf d' Promenad ging', bleibt s' das ganze Jahr zu Haus hocken und geht in einem spinatfarben Überrock herum.

LORENZ.- Sie taugt halt nur aufs Land. Sie will halt eine niedrige Person sein.

WURZEL.- Und doch redt s' hochdeutsch, und hat ihrs kein Mensch glernt (Raimund 2000: 19).

${ }^{13}$ Sus textos muestran un elevado nivel del dialectalidad. Se debe tanto al factor cuantitativo (índice de frecuencia de las marcas) como al factor cualitativo (presencia de marcas superficiales y profundas). Con ello se limita la comprensibilidad de las obras al ámbito de influencia del correspondiente dialecto.

${ }^{14}$ Horváth utiliza únicamente marcas superficiales, más de carácter coloquial que dialectal. Entre éstas destacan las partículas modales (Abtönungspartikeln), expresiones regiolectales y rasgos articulatorios generalizados en la lengua hablada. Véase ALBALADEJO (2006). 
HAVLITSCHEK.- (der Gehilfe Oskars, ein Riese mit blutigen Händen und ebensolcher Schürze, erscheint in der Türe der Fleischhauerei; er frißt eine kleine Wurst und ist wütend): Dummes Luder, dummes -

OSKAR.- Wer?

HAVLITSCHEK.- (deutet mit seinem langen Messer auf Ida): Das dort! Sagt das dumme Luder nicht, daß meine Blutwurst nachgelassen hat - meiner Seel, am liebsten tät ich sowas abstechen, und wenn es dann auch mit dem Messer in der Gurgel herumrennen müßt, wie die gestrige Sau, dann tät mich das nur freuen! (Horváth 1994: 11).

El texto de Reuter, escrito en bajo alemán, resulta prácticamente incomprensible para hablantes no familiarizados con esa variedad concreta. Esto se debe al empleo de gran número de marcas dialectales. En < seggen $>$, por ejemplo, se sustituye la vocal tónica y se duplica la consonante. Con ello se expresa la diferencia en calidad y cantidad vocálica en comparación con la lengua estándar. El poema de Hebel, escrito en un dialecto del alto alemán, también plantea dificultades de comprensión debido a la utilización de combinaciones grafemáticas no convencionales. Raimund, sin embargo, lleva a cabo la marcación sobre el inventario de la variedad escrita de la lengua estándar. Por tanto, las marcas, en su mayoría elisiones, no impiden la comprensibilidad suprarregional del texto. Finalmente, en Horváth se observan rasgos de dialectalidad de carácter superficial (elisiones generalizadas y léxico).

Karl Kraus, autor literario y editor de la revista Die Fackel, fue una de las voces más importantes que trató de rescatar la literatura de la gran época del teatro popular vienés, especialmente la de Nestroy, del confinamiento local-regional. Rechazó la equiparación de la obra de Nestroy con la de Anzengruber (autor de literatura dialectal y representante de la fase decadente del Wiener Volkstheater): "Nestroy, der kein österreichischer Dialektdichter, sondern ein deutscher Satiriker ist, ins Wienerische übersetzen[,] heißt ihm eine Anzengrube graben [...]" (Kraus 1925: 25).

Los prejuicios estéticos, derivados de un determinado purismo lingüístico tributario de una concepción tradicional del canon literario, han contribuido a generar una imagen distorsionada de esta literatura. Se ha simplificado y hasta negado su trascendencia como literatura marcadamente ficcional ${ }^{15}$ que refleja, desde un prisma satírico-crítico, los cambios sociológicos, económicos y culturales que conlleva la progresiva industrialización experimentada por Austria a lo largo del segundo tercio del siglo XIX. La crítica social y política presente en parte de las obras del Volkstheater adquiere un valor aún mayor habida cuenta del difícil contexto histórico en que se desarrolla, marcado por la existencia de la censura y de cierta represión policial, más patente en la época de Metternich ${ }^{16}$. Su confinamiento al medio local, producto de la errónea identificación como literatura dialectal ${ }^{17}$, y su devaluación injus-

\footnotetext{
${ }^{15}$ La ficcionalización es especialmente llamativa en relación con el subgénero de la pieza mágica (Zauberstück).

${ }^{16}$ De ahí que, frecuentemente, se recurra a la parodia para expresar esta actitud crítica sin correr el riesgo de ser prohibido.

${ }^{17}$ Con ello se activan, en nuestra opinión, mecanismos de rechazo o, al menos, de minimización.
} 
tificada como literatura meramente costumbrista sedimentada bajo la acumulación de estratos producto del paso del tiempo, la ha condenado a una pronunciada marginalidad. Aunque hoy en día se emitan juicios más matizados respecto del teatro popular vienés y no se califique, salvo en casos excepcionales como el de Wilpert, de forma expresa como literatura dialectal, implícitamente sigue considerándose como tal. Ésta es, al menos, la conclusión que cabe extraer, por ejemplo, del hecho de que Sowinski incluye a Nestroy y Raimund -junto con otros autores que tampoco merecen ser considerados escritores dialectales- en su Lexikon deutschsprachiger Mundartautoren. Sowinski justifica su decisión alegando un empleo del término "dialecto" en un sentido amplio de la palabra:

In bestimmten Fällen wurden auch Autoren berücksichtigt, die in schriftsprachlichen Literaturtexten mehr oder weniger intensiv mundartliche Eigenheiten einfügen, sei es im Wortschatz, im Satzbau, in den wörtlichen Reden oder als durchgehende Mischung von Schriftsprache und Mundart [...]. Gelegentlich finden sich auch Autoren mit konstruierten Mundarten oder mundartähnlichen Sprachen [...]. In allen diesen Fällen liegt ein erweiterter Mundartbegriff zugrunde [...] (Sowinski 1997: VI).

No obstante, también existen defensores de la postura opuesta. Entre los que discrepan de esta visión reduccionista y distorsionada acerca del teatro popular vienés se encuentran ilustres figuras de la cultura universal tales como Ludwig Wittgenstein, Ödön von Horváth, Thornton Wilder o Karl Kraus, por mencionar sólo los representantes más destacados.

\section{Origen e historia del Wiener Volkstheater}

Los inicios del teatro popular vienés se sitúan a principios del siglo XVIII. Es entonces cuando comienzan a fundarse los teatros de las periferias urbanas (Vorstadttheater), teatros de carácter comercial que dependían del gusto del público, en su mayoría perteneciente a la pequeña burguesía, que pagaba por ver las representaciones. En las periferias de grandes ciudades como Viena, Berlín, Múnich o Fráncfort, se establecen estos teatros comerciales, que se oponían a los teatros de la corte (Hof- und Residenztheater), los cuales estaban bajo la tutela del soberano y dependían del gusto y de los caprichos de la nobleza.

El contraste entre el Vorstadttheater y el Residenztheater se plasmaba en diferentes planos: mientras que en el Residenztheater (en el caso de Viena se trata del Burgtheater) se representaban dramas de carácter culto (temas mitológicos o históri$\cos$ ), escritos en un lenguaje elevado en verso, para un público noble y, generalmente, con una clara intención educativa (predominio del prodesse como función del teatro como Bildungseinrichtung), en el Vorstadttheater se imponían las piezas cómicas (comedias y farsas), escritas en un lenguaje, en prosa, adaptado a la extracción 
social de los personajes ${ }^{18}$ y que se representaban para el público burgués. El teatro popular pretendía, sobre todo, gustar a los espectadores. Se entiende, pues, que en sus obras se buscara, sobre todo, el entretenimiento de los asistentes a la función (predominio de la intención del delectare), tal y como lo expresa Nestroy, no sin cierta ironía:

G'fallen sollen meine Sachen, unterhalten, lachen sollen d'Leut, und mir soll die G'schicht a Geld tragen, dass ich auch lach', das is der ganze Zweck. G'spassige Sachen schreiben und nach dem Lorbeer trachten wollen, das is eine Mischung von Dummheit und Arroganz, das is grad so, als wie einer Zwetschgenkrampus' macht und gibt sich für einen Rivalen von Canova aus (Knight / Fabry 1967: 7).

Durante su primera fase, el teatro popular vienés muestra una influencia clara de la commedia dell'arte. Se trataba de un teatro de repentización (Stegreiftheater) donde los actores, sobre una base argumental preexistente, recurrían a la improvisación sobre el escenario. Su fundación se identifica con la figura de Josef Anton Stranitzky (1676-1726). Éste se hizo cargo en 1712 del Theater am Kärtnertor y, con ello, marcó el comienzo del teatro popular vienés ligado ya a un escenario estable, en contraposición al teatro ambulante anterior. Se constituye a partir de la confluencia de múltiples elementos e influencias teatrales, escénicas y musicales que convergen en Viena y que dan lugar a una peculiar literatura en lengua alemana con señas de identidad ${ }^{19}$ propias y claramente diferenciadas del resto de la literatura germana. Así, por ejemplo, Zeman (1996: 310) advierte:

Gegenüber allen anderen deutschsprachigen Ländern hatte sich hier ein theatralisch-literarischer Strom lebendig bewahrt, der zu dem Besten und Eigentümlichsten gehörte, was die gesamte deutschsprachige Literatur des 19. Jahrhunderts zu bieten hatte. Es war die zu ihrer Zeit bereits in ganz Mitteleuropa berühmte, durch viele Gastreisen - z. B. Raimunds und Nestroys - weithin bekannte Alt-Wiener volkstümliche Komödie und Posse.

La fenomenología del Wiener Volkstheater refleja múltiples influencias procedentes del teatro jesuita y escolar, de la ópera italiana y del teatro ambulante de tipo comedia de repentización. Su eclecticismo es el resultado de la confluencia de distintas manifestaciones artísticas -teatro, música, danza- y la utilización de todo tipo de medios escénicos que contribuían a aumentar la vistosidad del espectáculo. La fusión de todo ello sobre la base de una cultura barroca dio lugar a nuevas y peculiares formas teatrales.

A menudo, las piezas no eran creaciones originales sino que se trataba de reelaboraciones de otros textos literarios, tanto dramáticos como narrativos. Esas obras,

\footnotetext{
${ }^{18}$ De esta forma, los autores permitían al público reconocerse en las figuras tipo que actuaban sobre el escenario.

${ }^{19}$ El carácter identitario y peculiar del teatro popular vienés ha sido tratado en monografías y manuales a los que remitimos a los interesados en el tema. Véase, por ejemplo, HeIN (1997).
} 
que los autores del Wiener Volkstheater utilizaban como "cantera" para obtener tramas y personajes, generalmente carecían de auténtico valor literario, de ahí que casi todas hayan caído en el olvido. Es el caso, por ejemplo, de la farsa en un acto $A$ Day Well Spent, de John Oxenford, en la que se basa la farsa Einen Jux will er sich machen $^{20}$, una de las piezas más reconocidas de Johann Nestroy y que sirvió a Thornton Wilder de inspiración para su obra maestra The Matchmaker, la cual, a su vez, dio lugar al famoso musical Hello, Dolly.

Durante la primera fase del teatro popular vienés, representada por Stranitzky, Gottfried Prehauser (1699-1769) y Johann Joseph Felix von Kurz (1715-1784) se produce el apogeo de la comedia de repentización (Stegreifkomödie), en la que la figura cómica (el Hanswurst o el Bernardon) -una especie de bufón similar al Arlecchino de la commedia dell'arte- desempeña una función esencial. Mientras que este teatro gozaba del favor del público, la crítica lo consideraba peligroso debido a su carácter subversivo y poco edificante, pues, por encima de todo, pretendía entretener. En esa dirección apunta el siguiente comentario acerca de las obras de Nestroy:

[...] daß er seine Witze in den Bierkneipen auflese und seine Figuren nur den untersten Klassen entnehme. Derlei Klagen mögen auch dem Kaiser zugekommen sein, der am 26. Dezember 1834 [...] schrieb, es verlaute, daß das Theater an der Wien in der Achtung des Publikums immer tiefer herabsinke, indem daselbst Handlungen dargestellt werden sollen, die offenbar nicht nur der Schicklichkeit, sondern auch der Moralität zu nahe treten, wobei das Publikum Gefahr laufe, verkehrte Begriffe über menschliche und bürgerliche Lebensverhältnisse nach Hause zu tragen (Glossy 1933: 138).

Uno de los mayores detractores del teatro popular era Johann Christoph Gottsched (1700-1766). Con el fin de contribuir a elevar el nivel del teatro alemán abogaba, en su Escena alemana según las reglas y ejemplos de los antiguos (17411745), por la imitación de los modelos del teatro clasicista francés de Corneille, Molière y Racine, autores que habían escrito sus textos en consonancia con la Poéti$c a$ de Aristóteles a partir de una interpretación prescriptiva de la misma ${ }^{21}$. A Gottsched no sólo le preocupaba el gran poder subversivo de la figura cómica sino también la influencia, en su opinión, negativa, del uso de los dialectos. Estos constituían, según su punto de vista ${ }^{22}$, un obstáculo hacia una lengua alemana estandarizada que estuviera a la altura de otras lenguas como el francés.

${ }^{20}$ La importante historia de la recepción de la que goza esta obra en el ámbito anglosajón es, en nuestra opinión, una clarísima prueba del valor universal del teatro popular vienés de Nestroy y Raimund.

${ }^{21}$ Aristóteles había escrito su Poética con carácter descriptivo. Por tanto, los autores franceses realizaron una reinterpretación del texto aristotélico, convirtiendo los rasgos característicos de las piezas de la antigüedad en normas de obligado cumplimiento para la literatura de su época.

${ }^{22}$ Otros insignes representantes de las letras alemanas como Martin OpITZ (1597-1639), Georg SCHOtTEL (1612-1676) y Johann Christoph AdELUNG (1732-1806) se expresaron en este mismo sentido. 
Los escritos de Gottsched impulsaron en Austria el debate sobre la regularización del teatro (Wienerischer Hanswurst-Streit ${ }^{23}$ ). Ésta implicaba la prohibición de la figura del Hanswurst y la eliminación del dialecto en las obras. En 1751 se instauró en Austria la censura que obligaba a los autores a presentar los textos antes de la representación, se prohibieron la improvisación y las representaciones demasiado vulgares y, como consecuencia, desapareció la figura del Hanswurst.

Con la aprobación de la "libertad de espectáculo" (Spektakelfreiheit), en 1776 se reactiva nuevamente el teatro popular, que durante dos décadas había sufrido los efectos de la política de regularización de la comedia así como de la censura, y surgen numerosos teatros en los alrededores urbanos de Viena. Se inicia, así, la segunda fase del Wiener Volkstheater. Durante ésta se recupera, en parte, la tradición anterior a través de la creación de nuevas figuras bufonescas (el Kasperl ${ }^{24}$, el Thaddädl y el Staberl).

En este periodo se desarrollan, a partir de las distintas formas dramáticas anteriores, los dos géneros más importantes y característicos de la idiosincrasia peculiar del teatro popular vienés: la pieza mágica y la farsa ${ }^{25}$. Se trata de dos formas específicas en las que se manifiesta la pieza popular $\left(\right.$ Volksstück $\left.^{26}\right)$. La primera, la pieza mágica (Zauberstück), se caracteriza por la presencia de un mundo metafísico poblado por hadas, espíritus y magos. En él se desarrolla la acción secundaria que sirve de marco dramático a la acción principal, cuyo escenario es el mundo terrenal. La interacción entre ambos mundos se produce a través de la intervención de los seres sobrenaturales. Estos tratan de resolver los enredos generados en el mundo humano ${ }^{27}$, para lo cual emplean la "maquinaria" escénica del deus ex machina. Como recurso cómico, así como para facilitar el reconocimiento por parte del público, algunos de los seres sobrenaturales emplean el lenguaje local, algo que acentúa el carácter paródico de las obras.

El segundo género, la farsa (Posse), está marcado por el carácter popular de las piezas y una comicidad que pretende provocar la risa del público a través de la deformación de la realidad mediante una exageración más allá de los límites de lo

${ }^{23}$ Véase ZEMAN (1996: 280).

${ }^{24}$ Johann Joseph LA Roche (1745-1806) creó, en colaboración con Johann Matthias MENNINGER (1733-1793), la figura del Kasperl. Seguía desempeñando la función de figura cómica, ahora, sin embargo, sin lo obsceno (que caracterizaba al Hanswurst). A partir de mediados del siglo XIX, la figura del Kasperl adquiere gran relevancia dentro del teatro guiñol. Desde mediados del siglo XX se establece en Austria el Kasperltheater (el teatro guiñol con el Kasperl como figura central), sobre todo gracias a la televisión.

${ }^{25}$ HeIN (1997: 20): “Aus Zauberoper, Haupt- und Staatsaktion und Stegreifburleske entstehen die beiden für die Folgezeit des Volkstheaters wichtig werdenden Gattungen: Zauberspiel und Posse".

${ }^{26}$ Ibid., 71: "Volksstück meint im weitesten Sinne alle Produktionen des Volkstheaters; der Begriff selbst taucht, von frühen Belegen um 1800 abgesehen, erst um 1850 programmatisch auf. [...]. Volksstück kann ein Stück von dem, über das, für das Volk sein, es kann auf der thematischen Ebene Probleme des Volkes erfassen oder unterhaltend und belehrend auf das Volk wirken".

${ }^{27}$ A veces, la acción en el mundo humano parte de un suceso que ocurre en el mundo de las hadas, como, por ejemplo, en Der böse Geist Lumpazivagabundus oder Das liederliche Kleeblatt, de Johann Nestroy. 
verosímil y un humor que, en ocasiones, raya lo vulgar. Estas dos formas básicas generaron a su vez distintas variantes con una desigual incidencia según el periodo ${ }^{28}$.

Uno de los autores más destacados de esta fase, que se desarrolla entre 1780 1815, es Emmanuel Schikaneder ${ }^{29}$ (1751-1812), el libretista de La flauta mágica de Mozart. No es de extrañar, pues, que en La flauta mágica (genéricamente una Zauberoper) se reproduzca el típico esquema de los dos mundos, el terrenal y el de los seres sobrenaturales, de la pieza mágica.

La tercera fase del Wiener Volkstheater supone la culminación de esta manifestación literaria-teatral. Durante el segundo tercio del siglo XIX, el teatro popular vienés alcanza su máxima expresión artística a través de Johann Nestroy (18011862) y Ferdinand Raimund (1790-1836), ambos autores y actores a la vez. El hecho de que sus obras se sigan representando con asiduidad sobre los escenarios austriacos (también, aunque en menor medida, en Alemania y Suiza) deja constancia de que no se trata de una literatura desfasada y caduca, pues continúa fascinando al público contemporáneo así como a las nuevas generaciones de directores de teatro. En la Suiza alemana, sin ir más lejos, se han puesto en escena diversas obras de Nestroy y Raimund adaptadas al propio medio a través de versiones en Schwizerdütsch, y en Austria se celebran cada año los Nestroy-Spiele en la localidad de Schwechat. También a nivel internacional han adquirido cierta notoriedad gracias a que se han recibido algunos de sus textos, aunque se trate a todas luces de un número insuficiente ${ }^{30}$.

\section{EI dialecto en la literatura}

\subsection{Uso y función del dialecto en el texto literario}

Son muchos los autores literarios que a lo largo de la historia han recurrido en sus textos a lenguajes dialectales con el fin de que estos desempeñaran determinadas funciones dentro de sus obras. Grandes figuras de la literatura universal como William Shakespeare, Thomas Mann, Benito Pérez Galdos, Mark Twain o D. H. Lawrence han utilizado en ocasiones el dialecto. En las lenguas que muestran un elevado grado de dialectalización -como es el caso del alemán- se percibe una mayor incidencia de este recurso literario. Buena prueba de ello es la existencia del Lexikon

\footnotetext{
${ }^{28}$ El ejemplo más palpable de esa influencia cruzada se percibe en el género de la Zauberposse (farsa mágica) en el que se crea un híbrido a partir de la confluencia de ambos géneros.

29 HeIN (1997: 28): "Die vertraglich gebundenen Theaterdichter [...] vor allem Emanuel Schikaneder, Karl Friedrich Hensler, Joachim Perinet, Franz Xaver Karl Gewey und Ferdinand Kringsteiner, prägen mit der Entwicklung der verschiedenen Gattungen (Zauberspiel, Parodie, Lokalund Sittenstück, Singspiel, dramatisches Volksmärchen, Kasperliade) das Gesicht der zweiten großen Phase des Wiener Volkstheaters (1780-1815)".

${ }^{30}$ Destaca la recepción en lengua inglesa e italiana. También existen versiones francesas, portuguesas, checas, chinas, suecas, etc.
} 
deutschsprachiger Mundartautoren ${ }^{31}$ de Bernhard Sowinski, en el que se recopilan más de 10.000 autores sólo para el ámbito del alemán.

El hecho de que el lenguaje propio de la literatura sea una versión culta de la lengua estándar hace que los lenguajes no estándar (dialectos, lengua coloquial, tecnolectos, etc.) proporcionen rasgos específicos a los textos literarios. Además, estos se constituyen en recursos válidos para lograr la desautomatización del lenguaje dentro del texto literario, debido precisamente a que no forman parte del lenguaje literario canónico:

La introducción en el lenguaje literario del idioma habitual, del idioma regional o del término científico puede ser ya, sobre el fondo del lenguaje literario, un medio de modificar el sistema de señales; por así decirlo, una renovación de la señal que altera el estereotipo y obliga a esforzarse para comprender los hechos Šklovski (1971: 16).

La aparición de dialectos en la literatura genera contrastes intertextuales con otros textos escritos plenamente en un lenguaje estándar y/o intratextuales respecto de las partes redactadas en lengua estándar de los textos dialectalmente marcados.

Las funciones que desempeña el dialecto en la literatura varían dependiendo del género literario al que pertenece la obra, de los objetivos que se pretenden y también de la correspondiente época literaria ${ }^{32}$. Esto se debe a que el uso del dialecto ha ido adquiriendo diferentes connotaciones a lo largo de la historia y los motivos de su empleo obedecen a distintas razones. Otro factor a tener en cuenta a la hora de analizar sus funciones es si éstas tienen su reflejo en el seno de la obra (funciones endoliterarias) o si se proyectan más allá de ésta (funciones exoliterarias). Dado que en el teatro se imita la lengua hablada -y los dialectos son variedades esencialmente orales- es obvio que éste sea el género literario más proclive a utilizar los dialectos ${ }^{33}$.

Entre las funciones endoliterarias que cumplen los dialectos en los textos dramáticos y narrativos destaca la recreación naturalista del lenguaje de los personajes ${ }^{34}$, su identificación diatópica y diastrática y, en consecuencia, la estratificación de los caracteres dramáticos. A menudo también se suelen explotar determinados prejuicios - la supuesta menor capacidad intelectual de los hablantes de los dialectos frente a los hablantes de la lengua estándar-con fines cómicos ${ }^{35}$.

\footnotetext{
${ }^{31}$ Aunque en su mayoría se incluyan autores que practican literatura dialectal propiamente dicha, también aparecen autores cuyos textos sólo están marcados dialectalmente: no son obras redactadas íntegramente en dialecto y tampoco se trata de dialecto puro.

32 "Der Stilkontrast erhält in den verschiedenen literarischen Epochen und in den einzelnen Regionen durchaus unterschiedliche Funktionen" (HeIN 1983: 1629-1630).

${ }^{33}$ Ibíd., 1630: "Das Drama gilt überhaupt als "Vorreiter". Es hat zuerst Dialektsprecher in den Dialogen zugelassen [...]".

${ }^{34}$ En otras literaturas, el uso del dialecto puede adquirir funciones incluso ideológicas o políticas, tal y como se observa, por ejemplo, en el escritor y etnólogo peruano José María Arguedas.

35 "Während bei Rinckart die Mundart als einzige Ausdrucksform ihrer Sprecher ernsthaft gewürdigt wird, erscheint sie bei Gryphius und Grimmelshausen zugleich als Medium komischer
} 


\subsection{El dialecto en el Wiener Volkstheater}

Uno de los rasgos fundamentales del teatro popular vienés es la peculiar configuración lingüística que los autores confieren a los textos. En ellos se mezclan distintas variedades $^{36}$ y registros lingüísticos. El análisis del material lingüístico que aparece en los textos muestra una amplia gradación de la lengua ${ }^{37}$. La confluencia de personajes procedentes de distintas capas sociales explica el empleo de lenguajes que pueden oscilar entre registros cultos de la lengua estándar, diversos niveles lingüísticos identificables con la lengua coloquial y llegar hasta un cierto grado de dialectalidad ${ }^{38}$. De dialecto puro, sin embargo, no se puede (y no se debería) hablar.

Los siguientes fragmentos procedentes de la pieza mágica Der böse Geist Lumpazivagabundus oder Das liederliche Kleeblatt de Johann Nestroy muestran la variación intralingüística con la que se caracteriza a los distintos personajes:

FORTUNA.- Nun denn, so sei's! Ich will eine Bedingung setzen, die zugleich jenem Frechen, der meine Macht verspottet und glaubt, nur du (zu Amorosa) allein seist ihm gefährlich, das Gegenteil beweisen soll. - Ich wähle unter den Sterblichen drei seiner Anhänger, lockere Gesellen, jedoch solche, welche schon der Armut drückend Los gefühlt. Diese will ich mit Reichtum überschütten; werfen sie, wie er gesagt, das Glück zum Fenster hinaus, so dringe ich es ihnen zum zweiten Male wieder auf; treten sie es dann mit Füßen, so erkenne ich mich als besiegt, und Hilaris werde meiner Tochter Gemahl [...] (Nestroy 1999: 9-10).

LUMPAZIVAGABUNDUS.- Sie naht schon, die Mächtige, die mir oft meine fidelsten Brüderln entreißt! - Jetzt empfehl' ich mich! Aber noch einmal, Madame Fortuna, Sie fürcht' ich nicht; denn was meine wahren Anhänger sind, die machen sich nicht so viel aus Ihnen. Kommt's Glück einmal, so werfen sie's beim Fenster hinaus, und kommt's zum zweitenmal und will sich ihnen aufdringen auf eine

Wirkungen. Diese Funktion als Träger komischer Wirkungen, die zunächst aus dem Kontrast der Stände und der Sprachnormen, von vorgeblicher Bildung und scheinbarer Unbildung erwächst, hat die Mundart in der komischen Literatur bis in unsere Zeit beibehalten" SowINSKI (1997: XI).

${ }^{36} \mathrm{Si}$ bien es verdad que en algunas obras también se introducen otros dialectos alemanes -por ejemplo en Der böse Geist Lumpazivagabundus oder Das liederliche Kleeblatt aparecen el suabo y el bohemio-, en el teatro popular vienés la variedad habitual, junto con la lengua estándar, es por supuesto el dialecto vienés en distintas gradaciones.

${ }^{37}$ En la Deutsche Literaturgeschichte Band 6 (DTV) se describe la escritura de Nestroy de la siguiente manera: "Seine Texte schrieb er in einem der Schriftsprache angenäherten österreichischen Deutsch nieder, einer Mischung aus Hochsprache, Umgangssprache und Wiener Mundart" (VAN RINSUM: 263).

38 Zeman (1996: 343): "Nestroys Stücke und die mit ihnen verbundene satirische Komik erwachsen aus dem Wienerischen mit allen seinen Nuancierungen, die zwischen Hochsprache und breitem Dialekt denkbar sind". Habría que relativizar las palabras de Zeman respecto del "dialecto cerrado" (breiter Dialekt), pues las versiones escritas de los textos del teatro popular vienés muestran, en los pasajes más decididamente dialectales, siempre una cierta dilución del elemento dialectal, debido a la falta de convenciones gráficas para la trascripción del dialecto y la consiguiente eliminación, por parte de los autores, de algunos de sus rasgos. Aunque resulte más que probable que en la representación se volviese a una realización más cercana al dialecto no deja de ser cierto que los textos en sí no contienen un lenguaje fuertemente dialectal. 
dauerhafte Art, so treten sie's mit Füßen. - So behandeln meine echten Brüderln das Glück! - Gehorsamer Diener allerseits (Nestroy 1999: 8-9).

LEIM.- Da wär' ich beim Tor. Das is aber, so viel ich merk', eine ungefällige Stadt, denn wenn s' gefällig wär', so wär' s' mir auf halbem Weg entgegengekommen. Im Grund betracht't, ist's a Schand', ich bin ein ausgelernter Tischler, und es gehn mir ordentlich d'Füß aus ' $n$ Leim. Ist's denn aber auch anders möglich? Die Wirt' auf der Straßen haben ja Herzen so hart als ein Ast in ein' buchsbaumenen Pfosten. Woher kommt das aber? Weil die Leut' keine Bildung haben auf ' $n$ Land. Und warum haben s' auf 'n Land keine Bildung? [...] (Nestroy 1999: 11).

Frente al lenguaje estándar culto del hada Fortuna ${ }^{39}$, el espíritu maligno Lumpazivagabundus emplea un habla ligeramente coloquial $^{40}$ y el artesano Leim recurre a una variedad coloquial/dialectal de la lengua ${ }^{41}$.

La creación de contrastes intralingüísticos en el Wiener Volkstheater cumple diversas funciones. Por una parte, permite establecer diferencias diastráticas entre los distintos personajes ${ }^{42}$, insinuar distinciones intelectuales, marcar la procedencia geográfica de las figuras e introducir una expresión más natura$\operatorname{lista}^{43}$ frente a la idealización del lenguaje propia del Bildungstheater. También se explotan estas implicaciones para crear efectos cómicos, tal y como sostiene Rosenkranz (2007: 155):

${ }^{39}$ Prueba de ello son la declinación en dativo de "Male", cuando en los lenguajes coloquiales/dialectales esa terminación se elimina (generalmente, también en el estándar actual), o las estructuras "der Armut drückend Los" y "meiner Tochter Gemahl", donde se ha invertido el orden sintagmático normal (automatizado) del lenguaje común. El orden no marcado sería: "das drückende Los der Armut" y “der Gemahl meiner Tochter". La disposición sintagmática elegida apunta a un registro elevado (literario).

${ }^{40}$ El recurso de marcación esencial es la elisión (apócopes y aféresis), en algunos casos unida a la correspondiente contracción de los elementos lingüísticos adyacentes. Puntualmente también se dan marcas léxicas que expresan coloquialidad ("fidel" y "Brüderln").

${ }^{41}$ Hay una diferencia cuantitativa en el empleo de marcas entre el segundo y el tercer fragmento (mayor número de rasgos coloquiales/dialectales en el lenguaje de Leim), pero también una diferencia cualitativa en la marcación. El habla de Leim muestra rasgos más bien superficiales, como la elisión de la desinencia de la primera persona del singular del presente de indicativo ("ich wär', ich merk"'), aunque también se registran rasgos más claramente dialectales tales como el artículo indeterminado ("a Schand"” en lugar de "eine Schande"), el cual constituye una marca morfológica.

${ }^{42} \mathrm{La}$ estratificación del personal de la obra suele ser fundamental para el desarrollo de la trama de los textos del teatro popular vienés.

${ }^{43}$ Decimos "más naturalista", pues todo texto literario supone una "recreación artística" del lenguaje cotidiano. Es decir, que el lenguaje que aparece en una obra literaria siempre es, hasta cierto punto, artificial. De ahí que algunos investigadores se muestren contrarios a hablar de dialecto sin más cuando éste aparece en la literatura. Walter Schenker, por ejemplo, emplea el término "simulierter Dialekt" para dar cuenta de la modificación que sufren los lenguajes no estándar en un texto de carácter estético. SCHENKER (1977: 36): “[...] daß Dialekt da, wo er geschrieben wird, nicht mehr als Dialekt zu gelten hat. Wo also Dialekt in Literatur auftaucht, ist er grundsätzlich als lediglich simulierter Dialekt zu begreifen, und die Frage nach dem Verhältnis zwischen Dialekt und Literatur ist somit die Frage, wieweit und in welchen Weisen Literatur Dialekt adaptieren kann". 
Der Dialekt, obwohl an sich korrekt, kann einer gebildeten Schriftsprache gegenüber als inkorrekt erscheinen; die Komiker benutzen ihn daher zur Kontrastierung, wie Aristophanes, Shakespeare, Molière. Wie köstlich sind nicht der Kapitän Fluellen und der Pastor Evans von Shakespeare in ihren Dialekten gezeichnet! Das Schäferlied des letzten macht noch in Tiecks Übersetzung lachen.

En el teatro popular vienés, la función cómica es de esencial importancia dada la tipología de las piezas. En un sentido intratextual, en los diálogos la variedad local se convierte en el lenguaje propio de los protagonistas frente a la lengua estándar, que aparece como el auténtico elemento de contraste 44. No obstante, desde el punto de vista literario, el dialecto sigue siendo la variedad marcada y la lengua estándar es la variedad neutral.

\subsection{Identificación errónea con la literatura dialectal}

Una de las razones que explican la infravaloración y, en consecuencia, la escasa recepción del teatro popular vienés es la identificación (o equiparación) errónea del mismo con la literatura dialectal 45 :

Die Stücke der Volkstheater werden meist zu Unrecht unter der Rubrik "Mundartschauspiel" der Dialektdichtung zugeschlagen; in der Mehrzahl handelt es sich um vielschichtige sprachliche Gefüge, in denen Kontrastierungen von Dialekt und Hochsprache eine große Rolle spielen (z.B. bei Nestroy) (Hein 1983: 1628).

Esta equiparación conlleva una serie de implicaciones negativas. Teniendo en cuenta que el ámbito de difusión de la literatura dialectal suele estar delimitado por las fronteras lingüísticas de los dialectos (debido a los problemas de comprensión) se entiende que la definición de unas obras como dialectales condicione su recepción y valoración fuera del propio medio local o regional. A ello se suma la tendencia a minimizar la literatura dialectal debido a la supuesta limitación temática a la que los autores se debían someter, según algunas voces como, por ejemplo, la de Goethe46. De aplicarse ese principio, el ámbito de acción de la literatura escrita en una varie-

44 "Hier sind wir nun vollends im eigenen, nur der Posse zugehörigen Gattungsbezirk. Ihre Sprache ist die Mundart des Orts, an und von dem sie handelt. Mundart ist zugleich die unverwechselbare und unverzichtbare Verständigungsform der sozialen Schicht, die das Hauptpersonal der Posse stellt: der Kleingewerbetreibenden. [...] Angesichts der Weltsicht der Posse muß sie die Hochsprache aus mehreren Gründen als fremd und fernstehend empfinden: weil Hochsprache überall gilt, demnach der Eigenart und den Eigenbedürfnissen dieser bestimmten Menschen an diesem bestimmten Ort nicht gerecht wird" KLOTZ (1987: 136-137).

${ }^{45}$ Ya en el capítulo anterior hemos indicado los ejemplos de Wilpert y Sowinski que contribuyen a generar, como mínimo, una impresión de proximidad, que no se corresponde con la realidad textual.

46 "Goethe postuliert unter Berufung auf wirkungs- und gattungsästhetische Kategorien eine strikte thematische Einschränkung von Mundartpoesie [...]" (GRÄFE 2004: 60). El término Mundartpoesie no se refiere únicamente a la lírica sino a todas las obras literarias escritas en dialecto, también a obras dramáticas y narrativas. 
dad local o regional se reduciría considerablemente. En este sentido se expresa el lingüista Bruno Boesch, quien afirma que el dialecto sólo se puede emplear en el ámbito rural-campesino, por lo que no permite un gran desarrollo expresivo y creativo ${ }^{47}$. Siguiendo esa idea, el dialecto no permitiría la misma expresividad literaria de la que sí es capaz la lengua estándar. En consecuencia, la literatura dialectal no gozaría de la misma valoración estética que la literatura escrita en lengua estándar.

Las definiciones contemporáneas del término "literatura dialectal", como la de Klaus Mattheier ${ }^{48}$ o de Gero von Wilpert ${ }^{49}$, dejan claro que sólo aquellos textos redactados en su totalidad en una variedad local o regional cumplen los requisitos necesarios para que se pueda hablar de este tipo de literatura. En consecuencia, el Wiener Volkstheater no forma parte de la misma.

El hecho de que los textos de Raimund y Nestroy se publiquen en todos los territorios de lengua alemana en su versión original (sólo acompañados de un pequeño glosario con las expresiones opacas, debido a la diacronía o la diatopía) y no en versión estandarizada es prueba fehaciente de que no se trata de obras pertenecientes a la literatura dialectal. No hay que olvidar que uno de los rasgos distintivos de ésta es, precisamente, que carece de esa comprensión suprarregional que sí permiten los textos del teatro popular vienés.

De acuerdo con la definición de Mattheir, la literatura del Wiener Volkstheater se puede caracterizar como dialektgeprägte Literatur, es decir, "literatura dialectalmente marcada". Se trataría de unos textos escritos fundamentalmente en lengua estándar en los que se emplean marcas dialectales puntuales o incluso pasajes dialectales, ya sean discursos directos de los personajes dialectalmente marcados o rasgos dialectales del narrador ${ }^{50}$.

La escritura del lenguaje del teatro popular presenta unos problemas de reproducción, tanto léxica como, sobre todo, de realización. La articulación fonética y fonemática del lenguaje popular viene caracterizada, según Franz Xaver Gfirtner, por la reducción del número de sílabas pronunciadas, en comparación con la realización en lengua estándar ${ }^{51}$. A partir de los estudios que realizó, Gfirtner infirió la

${ }^{47}$ Véase GRÄFE (2004: 67).

48 "Geht man von der heute am weitesten verbreiteten Vorstellung von Mundartliteratur aus, so gehören dazu alle literarischen Texte, d. h. alle Texte, deren formale und inhaltliche Struktur nach fiktional-poetischen Prinzipien gestaltet ist, die als ganze durch eine nicht standardsprachliche, sondern nur in einer Teilregion verbreitete Sprachvarietät oder Merkmale dieser Varietät als literarisches und sprachliches Gestaltungsmittel charakterisiert ist" (MATTHEIER 1993: 635).

${ }^{49}$ Resulta contradictorio que Wilpert, por un lado, establezca como criterio para la literatura dialectal, que los textos estén redactados en su totalidad en dialecto y que, por otra parte, enumere, junto con escritores auténticamente dialectales, a otros (Nestroy, Raimund, Horváth, etc.) cuyos textos no cumplen esa premisa.

50 "Abzugrenzen von der Dialektliteratur im oben definierten Sinne ist die "dialektgeprägte Literatur", die in einen grundsätzlich standardsprachigen Rahmen bewußt oder unbewußt dialektale Merkmale oder Dialektpassagen einfügt, sei es als Zitate, als fiktive Sprachlichkeit der handelnden Personen oder als Dialektprägung der vom Erzähler verwendeten Standardsprache" (MATTHEIER 1993: 635).

${ }^{51}$ Desarrolló el Sprech-Niveau-Index que mide el nivel de habla, tomando como punto de partida la articulación estándar. El habla dialectal contaría con un número de sílabas articuladas muy inferior 
siguiente regla: la reducción silábica es tanto mayor cuanto más dialectal sea la articulación del hablante.

Aplicando esta herramienta de medición a los textos del Wiener Volkstheater se observa claramente que el lenguaje popular que en ellos aparece no puede clasificarse como dialectal, sino que debe considerarse como coloquial.

La trascripción gráfica de los dialectos supone un gran reto para los autores literarios debido a que no existe una codificación escrita de los mismos. Al no disponer de un código gráfico propio, los escritores que introducen variedades locales o regionales sólo pueden recurrir a la lengua estándar escrita. Como el único código gráfico normalizado existente no está pensado para recoger los rasgos peculiares del dialecto, muchos de estos se pierden si no se introducen nuevos elementos grafemáticos. El problema de estos, sin embargo, es que no suelen dejar de ser marcas idiolectales, porque carecen de la necesaria aceptación generalizada por parte de los hablantes, algo que limita su capacidad comunicativa.

A diferencia de lo que se observa en el caso de los textos de la literatura dialectal, en el teatro popular vienés no se emplea el procedimiento de crear nuevas combinaciones grafemáticas para reproducir con mayor fidelidad los rasgos propios del dialecto. Los siguientes fragmentos - uno procedente del teatro popular vienés (J. Nestroy) y el otro de la literatura dialectal (H. C. Artmann)- muestran las diferencias en el proceder de los autores a la hora de trascribir rasgos dialectales:

Ich hab Vierzehn Anzüg' theils licht und theils dunckel,

Die Frack und die Pantalon, Alles von Gunckel[.]

Wer mich anschaut, dem kommt das g'wiß nicht in Sinn,

$\mathrm{Da}$ ich trotz der Gard'rob ein Zerrissener bin.

Mein Gemüth is zerrissen[,] da is Alles zerstückt,

Und ein z'riss'nes Gemüth wird ein nirgends geflickt.

Und doch - müßt' i erklär'n wem den Grund von mein'n Schmerz,

So stundet ich da, als wie's Mandl beym Sterz;

Meiner Seel, 's is a fürchterlich's G'fühl,

Wenn man selber nicht weiß, was man will (Nestroy 1998: 12).

a dauwal miassad med an briaf

fon mia zu dia fliang

a schwäuwal miassed med an briaf

fon mia zu dia fliang

a r aumschl miassad med an briaf

fon mia zu dia fliang

a gaunze lufft fola fegl miassad kuma

und med mein briaf

fon mia zu dia fliang [...]

med ana rodn dintn how a da gschrim

das a de gean hob

al de la lengua estándar, mientras que el habla coloquial se situaría en una posición intermedia entre los extremos "lengua estándar-dialecto". Véase REIN (1983). 
med ana grinan dintn how a da gschrim

wia $r$ e no quoat hob auf dii -

med ana schwoazzn dintn owa mecht e da

jezt auf s weisse babia schreim

das ma r es heazz ausdroknt is

fua lauta woatn [...] (Artmann 1958: 34-35).

Mientras que el texto de Nestroy muestra un elevado nivel de comprensibilidad suprarregional debido a que la marcación dialectal se limita, básicamente, al ámbito de la articulación (destaca la elisión de la e-átona), la comprensión del poema de Artmann resulta muy difícil -incluso para personas familiarizadas con el dialecto vienés- dado que el autor trata de reproducir el valor fonético real del dialecto vienés. La adaptación que lleva a cabo Artmann no deja de ser una aproximación, pues muchos de los fonemas del vienés no se pueden representar de forma exacta con los grafemas del alemán estándar ${ }^{52}$. En algunos casos, Artmann crea nuevas combinaciones grafemáticas: por ejemplo en $<$ babia $>$ ( $<$ Papier $>$ en estándar), $<$ dia $>(<$ dir $>$ en estándar $) 0<$ schreim $>(<$ schreiben $>$ en estándar $)$.

\section{Conclusiones}

A día de hoy, el teatro popular vienés -y en concreto la obra de Nestroy y Raimund- cuenta con una percepción desigual en los distintos países de lengua alemana. La valoración tan positiva de la que gozan en Austria estos textos -y que ha llevado a su incorporación al canon nacional- contrasta con la posición periférica que siguen ocupando en los polisistemas suizo y alemán. Este hecho se refleja en las historias de la literatura, escritas a uno y otro lado de la frontera.

Son varios los factores que vienen condicionando negativamente la recepción y, sobre todo, la valoración de los autores. Al escaso interés de la comunidad investigadora por la literatura dialectal se suma el lógico desconocimiento de las numerosas variedades locales y regionales del alemán. Es, por tanto, de suponer que en muchos casos las opiniones vertidas sobre esta literatura sean producto de la simplificación. Son el resultado de una apreciación incompleta de la gradación de la marca dialectal tal y como se presenta en las mencionadas obras. En consecuencia, se borran las zonas de transición entre textos dialectales y dialectalmente marcados.

El presente estudio demuestra que tal equiparación contradice la realidad lingüística plasmada en los textos. Aunque es verdad que la marcación intralingüística introducida por los autores puede generar ciertas dificultades puntuales de comprensión, no es menos cierto que la incidencia de éstas se reduce a un mínimo gracias al aparato crítico de las ediciones modernas. Además, los textos cuentan

\footnotetext{
${ }^{52}$ Es el caso del fonema /a/ de las palabras de origen alemán (no afecta por tanto a los extranjerismos) que en los dialectos bávaros (y el vienés pertenece a ellos) adquiere un valor fonético que se sitúa entre [a] y [o]; de ahí que algunos lexicógrafos lo transcriban así: $<$ å $>$. Artmann, sin embargo, "simplifica" la transcripción mediante el empleo del grafema convencional $<0>$.
} 
con una trascripción gráfica muy cercana a la variedad escrita de la lengua estándar. No se plantea, pues, el problema derivado de los grafemas no convencionales $\tan$ habituales en las obras dialectalizadas.

Con la marcación intralingüística, un rasgo fundamental del teatro popular vienés, los autores pretenden establecer diferencias esencialmente de tipo sociocultural y, mediante este instrumento, caracterizar a los personajes ubicándolos en distintos estratos del continuo social. La tipología de las marcas -en su mayoría articulatorias- garantiza, en términos generales, la comprensibilidad total de los textos. Un análisis lingüístico en profundidad de la base textual permite, en nuestra opinión, recuperar las obras de su marcada e injustificada marginalización.

La peculiar configuración lingüística de los textos, una vez sometida a un estudio pormenorizado, y la universalidad de los temas elaborados prueba el error del supuesto carácter local de las obras.

La consideración negativa del elemento lingüístico, aun siendo sólo uno de los factores que ha condicionado la valoración de esta literatura, ha resultado ser determinante. La reevaluación del teatro popular vienés requiere la realización de un análisis objetivo, en los términos propuestos, que permita su reubicación dentro del canon de la literatura universal.

\section{Referencias bibliográficas}

ARTMAnN, H. C., med ana schwoazzn dintn. Salzburg: Otto Müller Verlag 1958.

AlBALADEJo, J. A., «El problema de los lenguajes periféricos en Ödön von Horváth», Hieronymus Complutensis 12 (2006), 63-67.

Beutin, W. et. al., Historia de la literatura alemana. Madrid: Cátedra 1991.

ECO, U., Historia de la fealdad. Barcelona: Lumen 2007.

Evan-Zohar, I., «Polysystemtheorie», en: Hagemann, S. (Hg.), Deskriptive Übersetzungstheorie. Berlin: Saxa Verlag 2009, 39-61.

Glossy, K., Wiener Studien und Dokumente. Zum 85. Geburtstag des Verfassers herausgegeben von seinen Freunden. Wien 1933, 138. Citado según HEIN (1997: 134).

GRÄFE, F., Dialektliteratur in Deutschland und Italien. Konstanz und Wandel von Bewertungsmustern. Marburg: Tectum Verlag 2004.

Hagemann, S., «Einleitung», en: Hagemann, S. (Hg.), Deskriptive Übersetzungsforschung. Berlin: Saxa Verlag 2009, 7-21.

HARrison, R., Wilson, K., «Introduction», en: Nestroy, J., Three Viennese Comedies. Columbia: Camden House 1986, 1-14.

HEIN, J., «Darstellung des Dialektsprechers in der neueren deutschen Dichtung», en: BESCH, W. et. al. (Hg.), Dialektologie. Ein Handbuch zur deutschen und allgemeinen Dialektforschung. Berlin / New York: Walter de Gruyter 1983, 1624-1636.

HeIn, J., Das Wiener Volkstheater. Darmstadt: Wissenschaftliche Buchgesellschaft 1997.

HeIN, J., «Vorwort zur dritten Auflage», en: HeIN, J., Das Wiener Volkstheater. Darmstadt: Wissenschaftliche Buchgesellschaft 1997, VII-VIII.

KLotz, V., Bürgerliches Lachtheater. Komödie - Posse - Schwank - Operette. Reinbek bei Hamburg: Rowohlt 1987. 
Knight, M., FABry, J., «Introduction», en: Nestroy, J., Three Comedies by Johann Nestroy. New York: Frederick Ungar Publishing Co. 1967, 1-27.

KraUS, K., «Nestroy und das Burgtheater», Die Fackel 676/78 (1925), 1-40.

MAtTHEIER, K. J., «Mit der Seele Atem schöpfen. Über die Funktion von Dialektalität in der deutschsprachigen Literatur», en: MATTHEIER, K. J. et. al. (Hg.), Vielfalt des Deutschen. Frankfurt am Main: Peter Lang 1993, 633-652.

NeSTROY, J., Der Zerrissene. Stuttgart: Reclam 1998.

NeSTROY, J., Der böse Geist Lumpazivagabundus oder Das liederliche Kleeblatt. Stuttgart: Reclam 1999.

REIN, K., «Bestimmende Faktoren für den variierenden Sprachgebrauch des Dialektsprechers», en: BESCH, W. et. al. (Hg.), op. cit. Berlin / New York: Walter de Gruyter 1983, 1443-1455.

Roetzer, H. G., Siguan, M., Historia de la literatura alemana. Barcelona: Editorial Ariel 1990.

RoSENKRANZ, K., Ästhetik des Häßlichen. Stuttgart: Reclam 2007.

SCHENKER, W., «Dialekt und Literatur», Zeitschrift für Deutsche Philologie 96 (1977), 34-48.

ŠKLOVSKI, V., Sobre la prosa literaria. Barcelona: Planeta 1971.

SOWINSKI, B., «Einleitung», en: SOWINSKI, B., Lexikon deutschsprachiger Mundartautoren. Hildesheim / Zürich / New York: Georg Olms Verlag 1997, V-XXV.

SumnER, I., «A Theory of Literary Dialect», en: WiLliAMSON, J. V. / BURKE, V. M. (eds.), A various language: Perspectives on American Dialects. New York: Holt, Rinehart \& Winston 1971, 145-177.

TrAmeR, E., «Preface», en: RAIMUnD, F., The Spendthrift. New York: Frederick Ungar Publishing Co. 1949, III-VII.

VAn Rinsum, A. y W., Deutsche Literaturgeschichte Band 6. München: dtv 1992.

WILPERT, G. v., Sachwörterbuch der Literatur. Stuttgart: Alfred Kröner Verlag 1989.

ZEMAN, H., «Die österreichische Literatur im ausgehenden 18. und im 19. Jahrhundert», en: ZEMAN, H. (Hg.), Literaturgeschichte Österreichs. Von den Anfängen im Mittelalter bis zur Gegenwart. Graz: Akademische Druck- und Verlagsanstalt 1996, 303-376. 